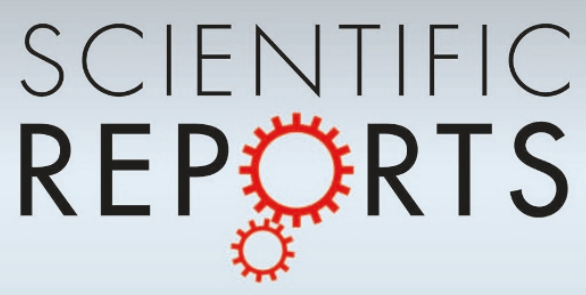

OPEN

SUBJECT AREAS:

SYNTHESIS OF

GRAPHENE

SURFACES, INTERFACES AND

THIN FILMS

Received

21 January 2014

Accepted

14 March 2014

Published

1 April 2014

Correspondence and requests for materials should be addressed to

X.-A.Z. (xazhang@ nudt.edu.cn) or L.W. (liwang@ncu.edu.cn)

* These authors contributed equally to this work.

\section{Growth of Millimeter-Size Single Crystal Graphene on Cu Foils by Circumfluence Chemical Vapor Deposition}

\author{
Chaocheng Wang ${ }^{1 *}$, Wei Chen ${ }^{2 *}$, Cheng Han ${ }^{3}$, Guang Wang ${ }^{2}$, Binbing Tang ${ }^{4}$, Changxin Tang ${ }^{4}$, \\ Yan Wang' ${ }^{1}$, Wennan Zou ${ }^{4}$, Wei Chen ${ }^{3}$, Xue-Ao Zhang ${ }^{2,5}$, Shiqiao Qin ${ }^{2,5}$, Shengli Chang ${ }^{2,5}$ \& Li Wang ${ }^{1,2,4}$
}

\begin{abstract}
'Department of Physics, Nanchang University, Nanchang 330031 , P.R. China, ${ }^{2}$ College of Science, National University of Defense Technology, Changsha 410073, P. R. China, ${ }^{3}$ Department of Chemistry, National University of Singapore, 3 Science Drive 3, Singapore $117542,{ }^{4}$ Institute for Advanced Study, Nanchang University, Nanchang 33003 1, P. R. China, ${ }^{5}$ State Key Laboratory of High Performance Computing, National University of Defense Technology, Changsha 410073, P. R. China.
\end{abstract}

A simply and reproducible way is proposed to significantly suppress the nucleation density of graphene on the copper foil during the chemical vapor deposition process. By inserting a copper foil into a tube with one close end, the nucleation density on the copper foils can be reduced by more than five orders of magnitude and an ultra-low nucleation density of $\sim 10$ nucleus $/ \mathrm{cm}^{2}$ has been achieved. The structural analyses demonstrate that single crystal monolayer graphene with a lateral size of $1.9 \mathrm{~mm}$ can be grown on the copper foils under the optimized growth condition. The electrical transport studies show that the mobility of such single crystal graphene is around $2400 \mathrm{~cm}^{2} / \mathrm{Vs}$.

raphene, a monolayer of carbon atoms arranged in a two-dimensional hexagonal lattice, was first isolated by mechanical exfoliation from $\mathrm{HOPG}^{1}$ and has shown extraordinary electronic properties for fundamental studies and potential applications in future electronics. Although various methods have been used to grow graphene on solid surfaces ${ }^{2-5}$, chemical vapor deposition (CVD) on metal foils is believed to be a promising way to produce large-area mono or few layer graphene $e^{6}$ However, a polycrystalline structure and a high density of domain/grain boundaries in typical graphene grown by CVD on metal foils cause variable electronic properties in difference areas and hinder the applications of graphene in devices ${ }^{7}$. It is still a challenge to grow large size singe crystal graphene to meet the requirements for large-scale production and integration of graphene-based electronic and optoelectronic devices.

In generally, reducing nucleation density is a crucial step to grow millimeter-size or larger size single crystal graphene by CVD. Nucleation mechanism for graphene grown on $\mathrm{Cu}$ foils by CVD is unclear so that many approaches have been explored to grow single crystal graphene with sub- or millimeter-size ${ }^{8}$. A long duration preannealing of copper foils with high flow or high pressure of $\mathrm{H}_{2}$ has been used to achieve millimeter-size single crystal graphene ${ }^{9}$. Suppressing evaporative loss of $\mathrm{Cu}$ from the foils during the CVD process is also suggested to a key point to fabricated $2 \mathrm{~mm}$ single crystal graphene ${ }^{10}$. Maintaining a $\mathrm{Cu}_{2} \mathrm{O}$ layer during the initial nucleation stage has been used to suppress the nucleation density to grow $5 \mathrm{~mm}$ single crystal graphene on $\mathrm{Cu}$ foils ${ }^{11-12}$

Here we report a simply and reproducible way to significantly suppress the nucleation density by slowing the gas flow close to the surface of the copper foil during the CVD process. The nucleation density on the copper foils can be reduced by more than five orders of magnitude and an ultra-low nucleation density of $\sim 10$ nucleus $/ \mathrm{cm}^{2}$ has been achieved by circumfluent CVD. Single crystal monolayer graphene with a lateral size of $1.9 \mathrm{~mm}$ and the mobility of $2400 \mathrm{~cm}^{2} / \mathrm{Vs}$ has been successfully grown on the copper foils under the optimized growth condition.

\section{Results}

Figure 1a shows the schematic drawing for the so called circumfluence CVD system used in this work. A small cuvette-like quartz tube ${ }^{13}$ with a diameter of $40 \mathrm{~mm}$ is inserted into a quartz tube in a typical low pressure CVD system which is pumped by an oil pump to maintain the pressure to be $100 \mathrm{~Pa}$. The close end of the inner tube highlighted as red in Fig. 1a faces the flow direction of the inlet mixture of $\mathrm{Ar}, \mathrm{H}_{2}$ and $\mathrm{CH}_{4}$. A copper foil for the growth of graphene is placed into the inner tube and near the close end of the inner tube. Flow field distribution within in the inner tube during the CVD process has been simulated by computational fluid dynamics (CFD) 

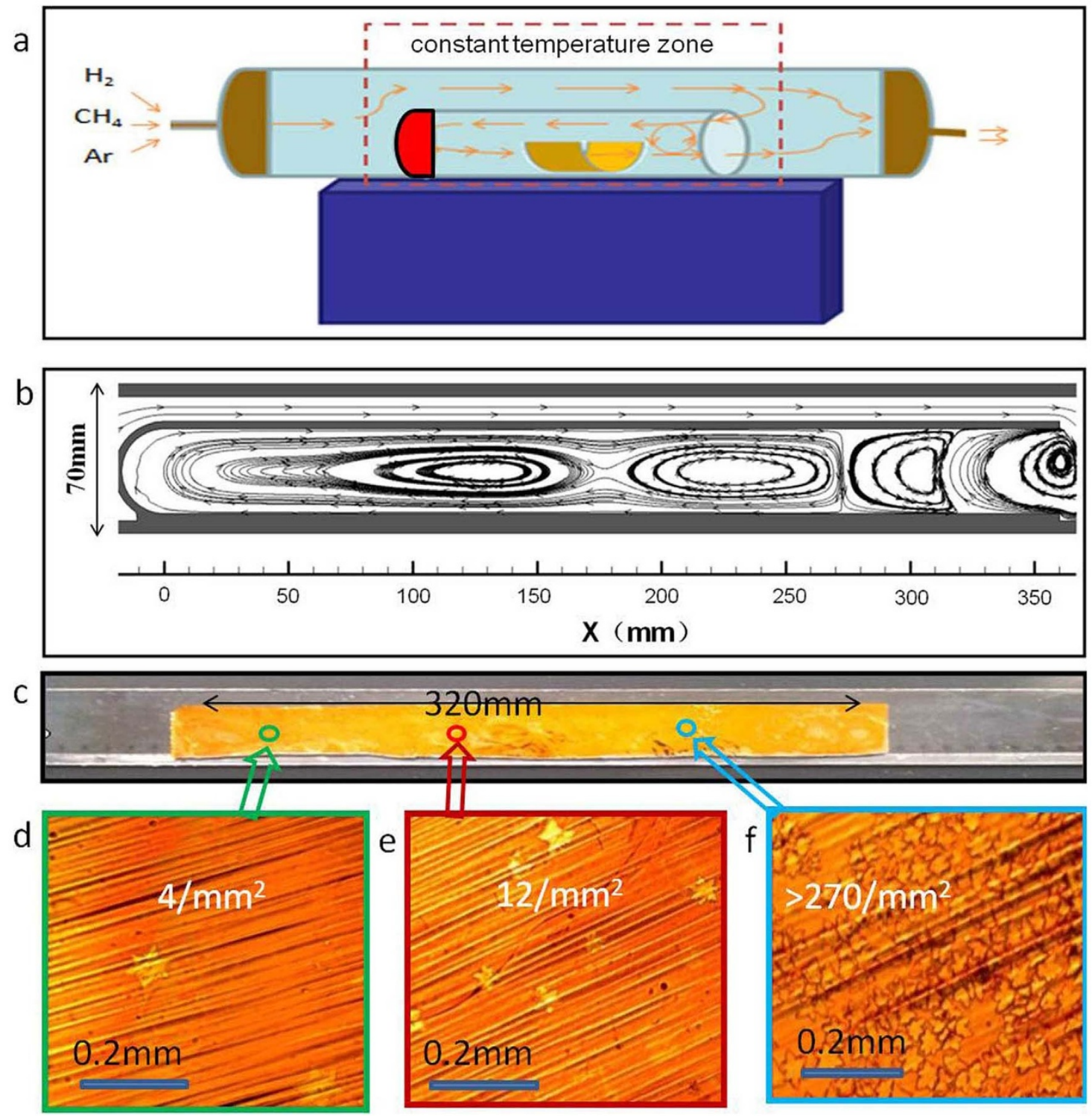

Figure $1 \mid$ (a) Schematic of home-built CVD system for synthesis of large-size graphene domains. The red dotted line frames constant temperature zone. The orange arrows indicate mixed gas flow (b). The streamline of mixed gas in inserted quartz tube from the simulation calculation.(c)A copper foil after CVD process.(d),(e).(f) Optical images of graphene domains on copper foil treated by $\mathrm{H}_{2} \mathrm{O}_{2}$.

method, as shown in Figure 1b. The mixture of the gases can not directly flow to the copper foil and form several vortices at the open side of the inner tube, which causes a circumfluent portion of the gases reach on the surface of the copper foil. Although the circumfluent gases are caused by the vortices, the analysis on the velocity of the gases reveals that the circumfluent gases close to the copper foil flow parallel with the surface of the copper foil and the velocity of the circumfluent gases can be reduced by 5 order magnitude compared with the velocity of the gases in the outer tube (Supplementary Figure S1).

Figure 1c gives an optical image of a copper foil with a length of $320 \mathrm{~mm}$ and a width of $20 \mathrm{~mm}$ after graphene was grown on the foil with the parameters: furnace temperature $1050^{\circ} \mathrm{C}, \mathrm{Ar}: \mathrm{H}_{2}: \mathrm{CH}_{4}=$ $100: 50: 0.1$ (in the unit of sccm) and the growth time of 15 mins. During the growth process, the left side of the foil was placed near the close end of the inner cuvette-like tube. The copper foil was treated by $\mathrm{H}_{2} \mathrm{O}_{2}$ to distinguish the graphene domains on the foil by optical microscopy ${ }^{14}$. The optical images for the marked areas (d, e and f) of the foil are shown in Fig. 1d, 1e and 1f, respectively. Each of the golden-bright starfish-like features in these optical images represent a graphene domain ${ }^{12}$. Although the graphene domains can be grown on the whole copper foil, there is a huge change on the density of graphene domain along the length direction of the copper foil. The density of graphene domains significantly increases from 4 domains/ $\mathrm{mm}^{2}$ in the area $\mathrm{d}$ on the left side of the foil near to the close end of the inner tube to 270 domains $/ \mathrm{mm}^{2}$ in the area fon the right side close to the open end of the tube. As the graphene domains are grown from the nuclei on the foil, the domain density directly reflects the nucleation density during the graphene growth process. Our observations suggest that the nucleation density on copper foil can be reduced to an ultra-low density of 4 nucleus $/ \mathrm{mm}^{2}$ (Supplementary Figure S2) in this designed circumfluence CVD, which gives the possibility for the fabrication of millimeter size graphene domains.

Figure 2a shows that a photograph of a copper foil after the growth of graphene with a ruler as a guide. The bright features in Fig. 2a represent the graphene domains while the yellow parts are the bare copper foil. All the graphene domains shown in the SEM images and the optical images appear elongated hexagonal shape with straight edges. The size of the largest graphene domain is larger than $1 \mathrm{~mm}$ (Fig. 2b) and this large graphene domain can be intactly transferred 
a

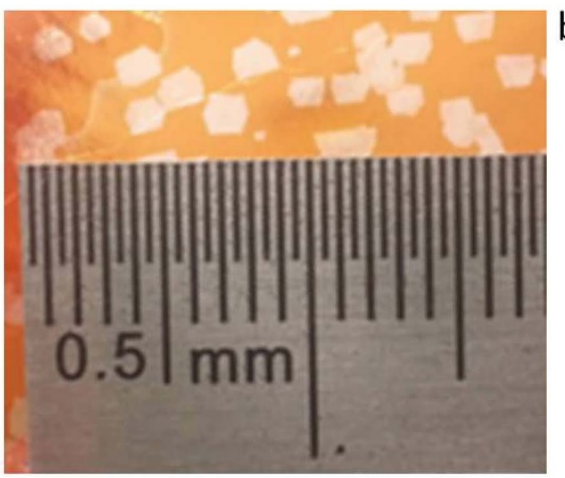

C

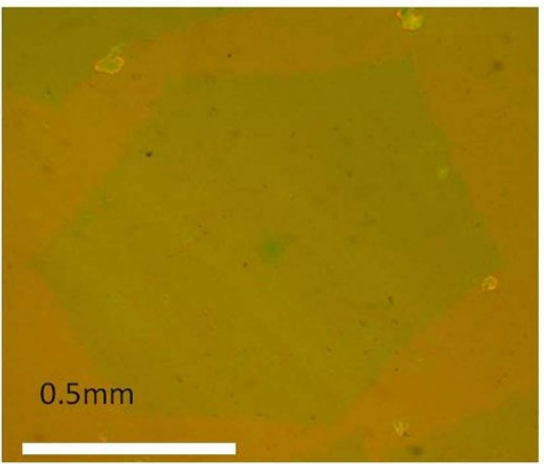

b

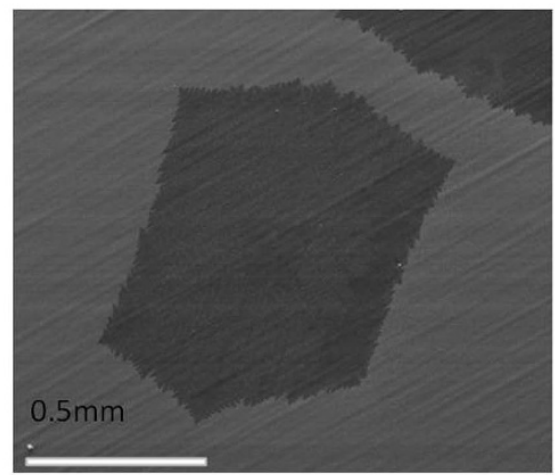

d

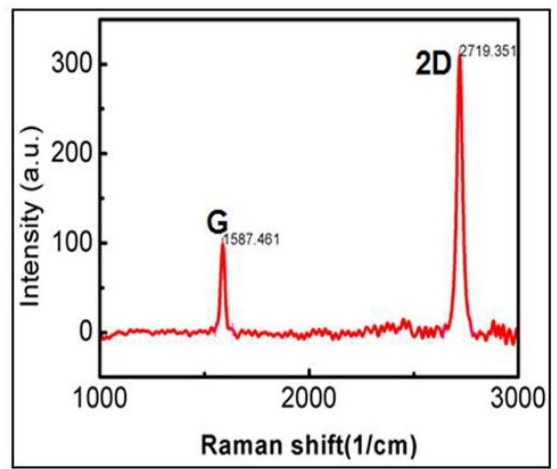

Figure $2 \mid$ (a), (b) Optical and SEM images of as-produced graphene domains on copper foil. (c) Optical image of one graphene domain transferred onto $\mathrm{SiO}_{2} / \mathrm{Si}$. (d) Raman spectra of graphene domain on $\mathrm{SiO}_{2} / \mathrm{Si}$.

from the copper foil to a $\mathrm{SiO}_{2} / \mathrm{Si}$ substrate (Fig. 2c). A typical Raman spectrum (Fig. 2d) taken on this graphene domain after being transferred on $\mathrm{SiO}_{2} / \mathrm{Si}$ substrate exhibits sharp $\mathrm{G}\left(1587 \mathrm{~cm}^{-1}\right)$ and $2 \mathrm{D}$ $\left(2719 \mathrm{~cm}^{-1}\right)$ peaks with a $\mathrm{G} / 2 \mathrm{D}$ ratio of 0.3 , which confirming that the graphene domains are single layer graphene sheets ${ }^{15}$. On the other hand, the fact that the disorder-induced $\mathrm{D}$ band $\left(1350 \mathrm{~cm}^{-1}\right)$ is hardly detected on such graphene domains further indicates the graphene domains are of high quality.

In order to characterize the crystalline of the millimeter-size graphene domains by transmission electron microscopy (TEM), the asgrown graphenes were transferred on an amorphous carbon-covered TEM grid. The SEM image of the graphene on TEM grid, Figure 3a, clearly shows that a $1.2 \mathrm{~mm}$-size hexagonal graphene domain was successfully transferred without any obvious damages to its structure. Figure $3 \mathrm{~b}$, the TEM image taken at the area highlighted as yellow frame in Fig. 3a, demonstrates the same edge structures as that observed by SEM, which allow us to determine the specific areas around this graphene domain with this edge as mark. Selected area electron diffraction (SAED) measurements were performed on 14 areas across this graphene domain to determine the crystalline. Figure $3 \mathrm{~d}-\mathrm{i}$ gives the six SAED patterns taken at the areas that are labeled correspondingly in Fig. 3a. The sharp and bright spots in all the SAED patterns fit the typical hexagonal structure well and are consistent with the previous reports ${ }^{11,12}$. The relative angle of graphene lattice derived from the SAED patterns on 14 areas shows less than $2^{\circ}$ rotation of the graphene lattice direction (Supplementary Figure S3), confirming that the millimeter-graphene domain is a single crystalline monolayer. Moreover, a domain visualization method based on polarized optical microscopy (POM) was also employed to confirm the crystallinity of the millimeter graphene domains $^{8,16}$. The transmission POM images taken at the liquid-crystal molecules (5CB) coated graphene domains on a quartz substrate (Supplementary Figure S4) show that the areas within each graphene domain are in similar colors, further confirming that there are no boundaries in the millimeter graphene domains, or in other words, these millimeter graphene domains are indeed single crystalline.

\section{Discussion}

The mobility measurements of graphene single crystal domain were performed on a back-gated field effect transistor (FET) built within a graphene domain on a highly doped p-type silicon substrate with $300 \mathrm{~nm}$ oxide. Figure 4a shows a photograph of the two-probe graphene FET with the length (L) and width (W) of the graphene channel of $10 \mu \mathrm{m}$ and $3 \mu \mathrm{m}$, respectively. Figure $4 \mathrm{~b}$ gives the transfer character curve of drain current $\left(\mathrm{I}_{\mathrm{ds}}\right)$ versus gate voltage $\left(\mathrm{V}_{\mathrm{g}}\right)$ in a vacuum chamber $\left(<10^{-6} \mathrm{~Pa}\right)$. The gate voltage was applied through the doped $\mathrm{Si}$ substrate and the source-drain bias $\left(\mathrm{V}_{\mathrm{ds}}\right)$ was constant at $0.1 \mathrm{~V}$. It manifests a positive shift in the Dirac point $\left(\mathrm{V}_{\text {Dirac }} \sim=+39 \mathrm{~V}\right)$, indicating that the graphene is highly $\mathrm{p}$-doped.

The mobility of the graphene domain was retrieved by fitting the transfer character curve to the equation ${ }^{17}$ :

$$
R_{\text {total }}=R_{c}+\frac{L}{W} \frac{1}{\mu_{F E}{\sqrt{e^{2} n_{0}^{2}+\left[C_{o x}\left(V_{g}-V_{\text {Dirac }}\right)\right.}}^{2}}
$$

where $R_{\text {total }}$ is the total resistance of the device including the channel resistance and contact resistance $R_{c}, e$ is the electron charge, $C_{o x}$ is the back-gate electrostatic capacitance, and $n_{0}$ is the carrier density due to residual impurities. A fitted curve is plotted in Figure $4 \mathrm{~b}$ by the dashed red line with the field effect electron mobility of $\sim 2440 \mathrm{~cm}^{2} \mathrm{~V}^{-1} \mathrm{~s}^{-1}$ and hole mobility of $\sim 1750 \mathrm{~cm}^{2} \mathrm{~V}^{-1} \mathrm{~s}^{-1}$ consistent with that of single-crystalline hexagonal grapheme flakes grown on a solid ${ }^{18-19}$ and liquid ${ }^{20} \mathrm{Cu}$ surface. The mobility of the millimeter graphene in our study is smaller than the previous reports ${ }^{9-11}$ for single crystalline graphene. It is well known that the interfacial properties between graphene and $\mathrm{SiO}_{2}$ have great influences on the mobility of the FET device. Therefore, we tentatively consider that the poor 
a
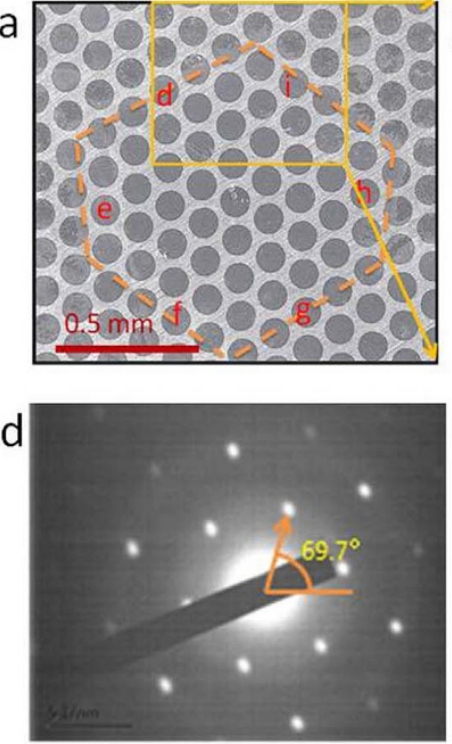

g

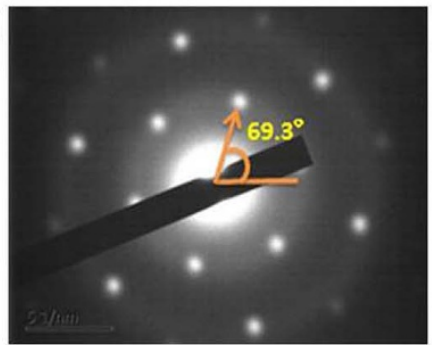


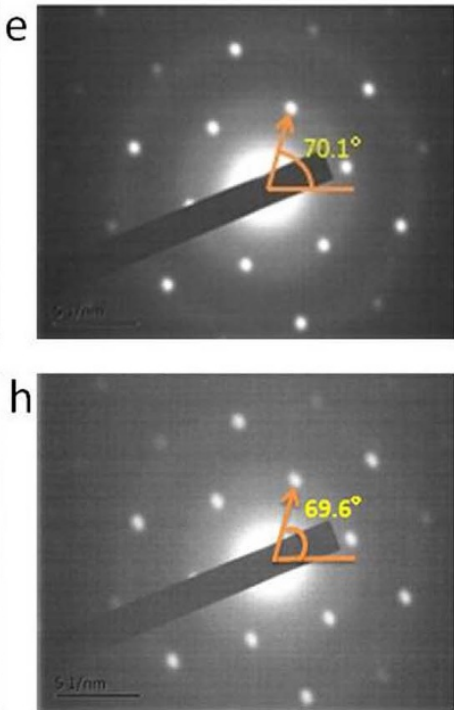
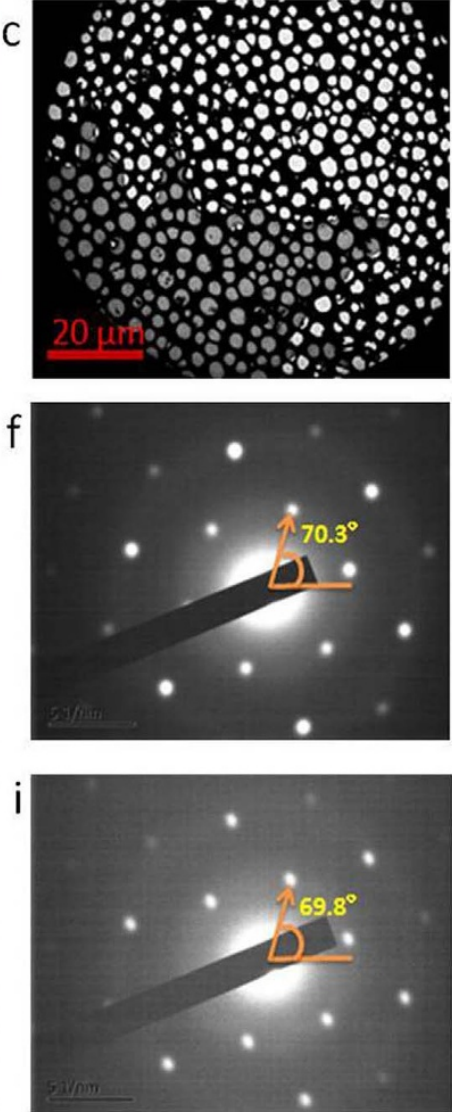

Figure $3 \mid$ (a), (b) SEM images of an as-produced graphene domain on an amorphous carbon-covered TEM grid. (c) TEM image of a corner of graphene domain. (d-f) SAED patterns correspond the areas in (a), small angle shifts may be due to the uneven copper grid surface.

interface between the graphene and the $\mathrm{SiO}_{2}$ in our study causes such small mobility of single crystal graphene.

In conclusion, the millimeter-size single crystal graphene domains have been fabricated on copper foils by the proposed circumfluent CVD method. The nucleation density of graphene on the copper foils



Figure $4 \mid$ Plot of drain current $\left(\mathrm{I}_{\mathrm{ds}}\right)$ versus gate voltage $\left(\mathrm{V}_{\mathrm{g}}\right)$ of graphene FET device measured at $300 \mathrm{~K}$. The collected data are plotted as a blue line, while the data fitted by red dash. The illustration is the optical image of the device. can be greatly reduced by controlling the gas flow distribution during the CVD process. The structural and electrical measurements confirm that the graphene obtained by this method is in single crystal and with highly electrical characteristic. The circumfluent CVD provides a simply and reproducible way to produce high quality single crystal graphene for large-scale integration of graphene device.

\section{Methods}

Graphene synthesis. A split tube furnace with a $40 \mathrm{~cm}$ heating zone and a $7 \mathrm{~cm}$ outer diameter quartz tube was used in a low-pressure CVD mode to grow graphene films on the $25 \mu \mathrm{m}$ thick Cu foil (99.8\%, Alfa Aesar, annealed, uncoated, item no. 46365). The copper foils were loaded into the home-built CVD system. The system was pumped down to a vacuum of $2 \mathrm{~Pa}$ in $10 \mathrm{~min}$ then filled with pure Argon, heated to $1050^{\circ} \mathrm{C}$ within $120 \mathrm{~min}$ with $50 \mathrm{sccm}$ of pure Argon. Then different molar ratio of $\mathrm{CH}_{4}(0.1-0.5 \mathrm{sccm}) / \mathrm{H}_{2}(25-100 \mathrm{sccm}) / \mathrm{Ar}(0-100 \mathrm{sccm})$ was introduced into the CVD system for the graphene growth at $1,050^{\circ} \mathrm{C}$ under variable pressure of 50 $150 \mathrm{~Pa}$ for $5 \mathrm{~min}-5 \mathrm{~h}$. After that the quartz tube was cooled in ambient environment.

Simulation of the flow field. Using the general computational fluid dynamics (CFD) software FLUENT, we analyzed the flow field of the crystallizer and obtain the whole flow solution including the pressure, velocity, temperature and density of the gases. The FLUENT, as an international popular CFD software, can simulate complex flows from incompressible to highly compressible. The streamline photograph of the crystallizer flow reflects the movement of the gases (the tangent direction of streamline is just the velocity direction of the local fluid). In our simulation, the gases were treated as a mixture and the flow was assumed to be steady.

Graphene transfer and mobility measurements. Graphene films were transferred onto p-type $\mathrm{Si}$ wafers with a $300 \mathrm{~nm}$ thick $\mathrm{SiO}_{2}$ layer using the same transfer method as previously ${ }^{21}$ and FET devices were fabricated using standard photolithography and electron beam lithography processes. The measurement was performed in the high vacuum of $\sim 10^{-6} \mathrm{~Pa}$ with a Keithley 4200 analyzer at room temperature, and before measurement devices were kept in the vacuum at least over night and treated by current annealing process to further desorb surface species on graphene.

Characterizations. The morphology and structure of the graphene were characterized with optical microscopy (SDC), scanning electron microscopy 
(FEI-Quanta 200F), transmission electron microscopy (JEOL JEM-2100 at $80 \mathrm{keV}$ ) and Raman spectroscopy (Buker SENTERRA, $514 \mathrm{~nm}$ laser wavelength).

1. Novoselov, K. S. et al. Electric field effect in atomically thin carbon films. Science 306, 666-669 (2004)

2. Emtsev, K. V. et al. Towards wafer-size graphene layers by atmospheric pressure graphitization of silicon carbide. Nature Mater. 8, 203-207 (2009)

3. Kim, K. S. et al. Large-scale pattern growth of graphene films for stretchable transparent electrodes. Nature 457, 706-710 (2009).

4. Li, X. et al. Large-area synthesis of high-quality and uniform graphene films on copper foils. Science 324, 1312-1314 (2009).

5. Gao, L. et al. Repeated growth and bubbling transfer of graphene with millimetresize single-crystal grains using platinum. Nature Commun. 3, 699 (2012).

6. Bae, S. et al. Roll-to-roll production of 30-inch graphene films for transparent electrodes. Nature Nanotech. 5, 574-578 (2010).

7. Wei, D. \& Liu, Y. Controllable synthesis of graphene and its applications. Adv. Mater. 22, 3225-3241 (2010).

8. Wang, H. et al. Controllable synthesis of submillimeter single-crystal monolayer graphene domains on copper foils by suppressing nucleation. J. Am. Chem. Soc. 134, 3627-3630 (2012).

9. Yan, Z. et al. Toward the synthesis of wafer-scale single-crystal graphene on copper Foils. ACS Nano. 6, 9110-9117 (2012).

10. Chen, S. et al. Millimeter-size single-crystal graphene by suppressing evaporative Loss of $\mathrm{Cu}$ During low pressure chemical vapor deposition. Adv. Mater. 25, 2062-2065 (2013).

11. Zhou, H. et al. Chemical vapour deposition growth of large single crystals of monolayer and bilayer grapheme. Nature Commun. 4, 2096 (2013).

12. Hao, Y. et al. The role of surface oxygen in the growth of large single-crystal graphene on Copper. Science 342, 720-723 (2013).

13. Fan, L. L. et al. Controllable growth of shaped graphene domains by atmospheric pressure chemical vapour deposition. Nanoscale 3, 4946 (2011).

14. Jia, C., Jiang, J., Gan, L. \& Guo, X. Direct optical characterization of graphene growth and domains on growth substrates. Sci. Rep. 2, 707 (2012).

15. Ferrari, A. C. et al. Raman spectrum of graphene and graphene layers. Phys. Rev. Lett. 97, 187401 (2006)

16. Kim, D. W., Kim, Y. H., Jeong, H. S. \& Jung, H. T. Direct visualization of large-area grapheme domains and boundaries by optical birefringency. Nature Nanotech. 7 , 29-34 (2012).

17. Suk, J. W. et al. Enhancement of the Electrical Properties of Graphene Grown by Chemical Vapor Deposition via Controlling the Effects of Polymer Residue. Nano. Lett. 13, 1462-1467 (2013).

18. Wu, B. et al. Equilangular hexagon-shape-controlled synthesis of graphene on copper surface. Adv. Mater. 23, 3522-3525 (2011).
19. Wu, W. et al. Growth of single crystal graphene arrays by locally controlling nucleation on polycrystalline $\mathrm{Cu}$ using chemical vapor deposition. Adv. Mater. 23, 4898-4903 (2011).

20. Geng, D. et al. Uniform hexagonal graphene flakes and films grown on liquid copper surface. PNAS 109, 7992-7996 (2012).

21. Chan, J. et al. Reducing extrinsic performance limiting factors in graphene grown by chemical vapor deposition. ACS Nano. 4, 3224-3229 (2012).

\section{Acknowledgments}

This work was financially supported by National Key Basic Research Program of China (No 2013CB934200). L.W. acknowledges the Program for New Century Excellent Talents in University, Ministry of Education of China (NCET -11-1003) and Jiangxi Provincial “Ganpo Talentes 555 Projects". X.Z. acknowledges the open foundations of the innovation platform of Hunan Key Laboratories (No. 13K022) and State Key Laboratory of High Performance Computing (No. 201301-02)

\section{Author contributions}

L.W. and X.Z. conceived and designed the experiments. C.W. and W.C. co-performed the experiments. C.H., G.W., B.T. and W.C. contributed analysis tools and assisted the experiments. C.T. and W.Z. carried out simulate calculations. Y.W. helped to prepare Supplementary Figure S2. L.W., S.C. and S.Q. supervised the project. C.W., W.C. and L.W. co-wrote the paper. All authors discussed the results and analyzed the data and commented on the manuscript.

\section{Additional information}

Supplementary information accompanies this paper at http://www.nature.com/ scientificreports

Competing financial interests: The authors declare no competing financial interests.

How to cite this article: Wang, C.C. et al. Growth of Millimeter-Size Single Crystal Graphene on Cu Foils by Circumfluence Chemical Vapor Deposition. Sci. Rep. 4, 4537; DOI:10.1038/srep04537 (2014).

This work is licensed under a Creative Commons Attribution-NonCommercialShareAlike 3.0 Unported License. The images in this article are included in the article's Creative Commons license, unless indicated otherwise in the image credit; if the image is not included under the Creative Commons license, users will need to obtain permission from the license holder in order to reproduce the image. To view a copy of this license, visit http://creativecommons.org/licenses/by-nc-sa/3.0/ 